bioRxiv preprint doi: https://doi.org/10.1101/2020.04.10.036343; this version posted April 14, 2020. The copyright holder for this preprint (which

was not certified by peer review) is the author/funder, who has granted bioRxiv a license to display the preprint in perpetuity. It is made available under aCC-BY-NC-ND 4.0 International license.

\title{
CoV Genome Tracker: tracing genomic footprints of Covid-19 pandemic
}

Saymon Akther ${ }^{1,2}$, Edgaras Bezrucenkovas², Brian Sulkow², Christopher Panlasigui' ${ }^{2}$, Li Li ${ }^{1,2}$, Weigang $\mathrm{Qiu}^{1,2,3, *}$, and Lia $\mathrm{Di}^{2, *}$

${ }^{1}$ Graduate Center, City University of New York, USA; ${ }^{2}$ Department of Biological Sciences, Hunter College, City University of New York, New York, New York 10065, USA; ${ }^{3}$ Department of Physiology and Biophysics \& Institute for Computational Biomedicine, Weil Cornell Medical College, New York, New York 10021, USA

Emails: Saymon Akther < sakther@gradcenter.cuny.edu>; Edgaras Bezrucenkovas <edgaras993@gmail.com>; Brian Sulkow<drtwisto@gmail.com>; Christopher Panlasigui <christopher.panlasigui@gmail.com>; Li Li<lli4@gradcenter.cuny.edu>; *Co-correspondence: Weigang Qiu <weigang@genectr.hunter.cuny.edu> \& Lia Di <dilie66@gmail.com>

\begin{abstract}
Summary: Genome sequences constitute the primary evidence on the origin and spread of the 2019-2020 Covid-19 pandemic. Rapid comparative analysis of coronavirus SARS-CoV-2 genomes is critical for disease control, outbreak forecasting, and developing clinical interventions. CoV Genome Tracker is a web portal dedicated to trace Covid-19 outbreaks in real time using a haplotype network, an accurate and scalable representation of genomic changes in a rapidly evolving population. We resolve the direction of mutations by using a bat-associated genome as outgroup. At a broader evolutionary time scale, a companion browser provides geneby-gene and codon-by-codon evolutionary rates to facilitate the search for molecular targets of clinical interventions.
\end{abstract}

Availability and Implementation: CoV Genome Tracker is publicly available at http://cov.genometracker.org and updated weekly with the data downloaded from GISAID (http://gisaid.org). The website is implemented with a custom JavaScript script based on jQuery (https://jquery.com) and D3-force (https://github.com/d3/d3-force).

Contact: weigang@genectr.hunter.cuny.edu, City University of New York, Hunter College Supplementary Information: All supporting scripts developed in JavaScript, Python, $\mathrm{BASH}$, and PERL programming languages are available as Open Source at the GitHub repository https://github.com/weigangq/cov-browser. 


\section{Usages \& Innovations}

Genomic epidemiology comparatively analyzes pathogen genome sequences to uncover the evolutionary origin, trace the global spread, and reveal molecular mechanisms of infectious disease outbreaks including the latest coronavirus pandemic caused by the viral species SARSCoV-2 (1-4). The unprecedented public-health crisis calls for real-time analysis and dissemination of genomic information on SARS-CoV-2 isolates accumulating rapidly in databases such as GISAID (http://gisaid.org) (5,6). To meet the challenge of real-time comparative analysis of SARS-CoV-2 genomes, we developed the CoV Genome Tracker (http://genometracker.org) with a supporting bioinformatics pipeline. Key features of the CoV Genome Tracker include interactive visualization and exploration of geographic origins, transmission routes, and viral genome changes of Covid-19 outbreaks (Fig 1). A companion comparative genomics website displays the 2003-2004 SARS-CoV and the 2019-2020 SARS-CoV-2 outbreaks in the evolutionary context of their wildlife relatives $(1,7)$.

At the micro-evolutionary time scale, a key distinction of CoV Genome Tracker from the Nextstrain Covid-19 browser (https://nextstrain.org/ncov) (6) is our adoption of a haplotype network - instead of a phylogenetic tree - as the analytic framework as well as the visual guide (Fig 1). A haplotype network offers several advantages over a phylogenic tree. First, at the time scale of days and months, loss and fixation of alleles are rare and the ancestral and descendant genotypes are both present in the population. As such, tree-based phylogenies can be misleading because tree-based phylogenetic algorithms compel all sampled genomes into leaf nodes regardless of ancestral or descendant genotypes, meanwhile introducing hypothetical ancestors as internal nodes. Second, phylogenetic reconstruction typically assumes a mutation-driven process with complete lineage sorting. Violation of these assumptions results in misleading evolutionary relations, for example, when recombination is present or when genes remain polymorphic $(8,9)$. Third, a haplotype network requires less abstract comprehension of evolutionary processes than a phylogenetic tree does. For example, edges of a haplotype network depict genetic changes from a parent to a descendant genome, while branches of a phylogenetic tree represent

60 Fourth, a haplotype network is more scalable than a phylogenetic tree as a visual tool. This is

61 because the total number of nodes of a phylogenetic tree grows linearly with the number of 62 genomes, resulting in a crowded visual space. In contrast, additional genomes add to the size 
63 but not the total number of nodes of a haplotype network if they share the same haplotype se-

64 quence with previously sampled genomes.

A further innovation of the haplotype network used in the CoV Genome Tracker is the inclusion of an outgroup genome to polarize all mutational changes. Conventional haplotype networks show mutational differences but not mutational directions on edges (10-12). The directed haplotype network in CoV Genome Tracker is thus informative for tracing the origin, following the spread, and forecasting the trend of Covid-19 outbreaks across the globe (Fig 1). To date, one published study and two preprint manuscripts use haplotype networks to represent the genealogy of SARS-CoV-2 isolates (13-15). These networks are however based on a much smaller number of genomes, non-interactive, and non-directional.

At the macro-evolutionary time scale, CoV Genome Tracker provides more in-depth fea-

74 tures than the Nextstrain browser on SARS-CoV-2 genome evolution

75 (https://nextstrain.org/groups/blab/sars-like-cov) (6) (Supplemental Fig S1). Modeled after Bor76 reliaBase (http://borreliabase.org), a browser of Lyme disease pathogen genomes (16), the com-

77 parative genomics browser of CoV Genome Tracker provides analytical features including sequence alignments, gene trees, and codon-specific nucleotide substitution rates. As such, the macro-evolutionary browser facilitates exploring the wildlife origin of SARS-CoV-2, identifying functionally important gene sites based on sequence variability, and understanding mechanisms of genome evolution including mutation, recombination and natural selection $(3,4)$.

\section{Methods \& Implementation}

The micro-evolutionary and macro-evolutionary browsers of the CoV Genome Tracker are continuously updated according to the following workflows.

For the Covid-19 genome browser, we download genomic sequences and associated metadata of SARS-CoV-2 isolates from GISAID (5), which are subsequently parsed with a PY-

87 THON script ("parse-metadata.ipynb"; all scripts available in GitHub repository http://cov.ge-

88 nometracker.org). We use a custom BASH script ("align-genome.sh") to align each genome to 89 an NCBI reference genome (isolate Wuhan-Hu-1, GenBank accession NC_045512) with Nu90 cmer4 (17), identify genome polymorphisms with Samtools and Bcftools (18), and create a hap-

91 lotype alignment using Bcftools. To minimize sequencing errors, we retain only phylogenetically 92 informative bi-allelic single-nucleotide polymorphism (SNP) sites where the minor-allele nucleo93 tide is present in two or more sampled genomes. To maximize network stability, a custom Perl 
94 script ("impute-hap.pl") is used to trim SNP sites at genome ends where missing bases are common, discard haplotypes with more than 10\% missing bases, (optionally) impute missing bases of a haplotype with homologous bases from a closest haplotype (19), and identify unique haplotypes using the BioPerl package Bio::SimpleAlign (20). To root the haplotype network, we include the genome of a closely related bat isolate (RaTG13, GenBank accession MN996532) (1) as the outgroup (using however only nucleotides at the SNP sites present among human isolates).

We use two methods to infer a network genealogy of unique haplotypes. In one approach, 102 we infer a maximum parsimony tree using the DNAPARS program of the PHYLIP package (21). 103 A custom Perl script ("hapnet-pars.pl") transforms the resulting maximum parsimony tree into a 104 phylogenetic network by replacing internal nodes with the nearest haplotypes where tree dis105 tances between the two are zero. Alternatively, we use a custom Perl script ("hapnet-mst.pl") to 106 reconstruct a minimum-mutation network of unique haplotypes based on the Kruskal's minimum 107 spanning tree (MST) algorithm implemented in the Perl module Graph (https://metacpan.org/re108 lease/Graph). Both Perl scripts polarize the edges of the haplotype network according to the 109 outgroup sequence by performing a depth-first search using the Perl module Graph::Tra110 versal::DFS (https://metacpan.org/pod/Graph::Traversal::DFS). The Perl scripts output a di111 rected graph file in the JavaScript Object Notation (JSON) format. The JSON network file is read 112 by a custom JavaScript, which layouts the website with the JavaScript library jQuery 113 (http://jquery.com) and creates an interactive force-directed rendering of the haplotype network with the JavaScript library D3-force (http://d3js.org).

For the comparative genomics browser of CoV, we download genomes of a human-host SARS-CoV-2 (isolate WIV2, GenBank accession MN996527), a human-host SARS-CoV (isolate 117 GD01, GenBank accession AY278489), and closely related coronavirus isolates from bat hosts 118 from the NCBI Nucleotide Database. We extract coding sequences from each genome and iden119 tify orthologous gene families using BLASTp (22). For each gene family, we obtain a codon 120 alignment using MUSCLE and Bioaln $(23,24)$. We reconstruct maximum-likelihood trees for in121 dividual genes as well as for the whole genome based on a concatenated alignment of ten genes 122 using FastTree (25). For each gene, we estimate the maximum-parsimony number of nucleotide 123 changes at each codon position using DNACOMP of the PHYLIP package (21). Differences in 124 nucleotide substitution rates between the predominantly synonymous $3^{\text {rd }}$ codon position and the 125 other two codon positions are indicative of forces of natural selection. For example, a higher 126 substitution rate at the $3^{\text {rd }}$ codon position than the rate at the $1^{\text {st }}$ and $2^{\text {nd }}$ positions indicates 
127 purifying selection while a higher or similar rate at the $1^{\text {st }}$ and $2^{\text {nd }}$ codon positions relative to the 128 rate at the $3^{\text {rd }}$ codon position suggests adaptive diversification (e.g., at the Spike protein-encod-

129 ing locus) (2). The CoV comparative genomics browser is developed with the same software 130 infrastructure supporting BorreliaBase (http://borreliabase.org), a comparative genomics 131 browser of Lyme disease pathogens (16).

In summary, the CoV Genome Tracker facilitates up-to-date and interactive analysis of viral genomic changes during current and future coronavirus outbreaks. The CoV Genome Tracker uses a haplotype network, a more accurate and scalable model than a phylogenetic tree to analyze and visualize genomic changes in the rapidly evolving SARS-CoV-2 population (6).

137 We improved upon conventional haplotype networks by resolving the direction of mutational 138 changes based on an outgroup genome $(10,12)$. Future development will include implementing 139 probabilistic network algorithms such as maximum parsimony probability $(10,11)$, developing 140 methods for testing network accuracy and stability, analyzing association between genomic 141 changes and network characteristics (e.g., association between the number of nonsynonymous 142 mutations and the in- and out-degrees of nodes), performance optimization, usability improve143 ments, and incorporating a mechanism for community feedback.

\section{Declaration}

Availability of website \& source codes

CoV Genome Tracker is publically available at http://cov.genometracker.org. All source codes are released as Open Source and available at https://github.com/weigangq/cov-browser.

148 The repository contains BASH, Perl, Python, R, and JavaScript codes for data processing pipe149 line, network reconstruction, and web development.

\section{Authors' Contributions}

151 S.A. implemented the genome processing pipeline and drafted the manuscript. E.B. de152 veloped the workflow for downloading and parsing data from the GISAID database. B.S. per153 formed network stability analysis and contributed to website design. C.P. prepared and main154 tains online documentation. L.L. contributed to network analysis, drafting manuscript, and online 155 documentation. W.Q. conceived the project, developed and implemented the network algorithm, 156 and drafted the manuscript. L.D. developed the meta-data pipeline, designed the website, im157 plemented JavaScript codes, and prepared the figures. 
158

159

160

161

162

163

\section{Acknowledgements}

We gratefully acknowledge the authors, originating and submitting laboratories of the sequences from GISAID's EpiCoV ${ }^{\mathrm{TM}}$ Database on which this research is based. All submitters of data may be contacted directly via www.gisaid.org. We thank Desiree Pante, Bing Wu, and Ramandeep Singh for participation in data entry. We thank Dr Yozen Hernandez for system administration of computer networks. We thank Jonathan Sulkow for contributing to webpage design.

\section{Funding}

S.A. and L.L. are supported in part by the Graduate Program in Biology from the Graduate Center, City University of New York. This work was supported in part by the National Institute of Allergy and Infectious Diseases (NIAID) (AI139782 to W.Q.) of the National Institutes of Health (NIH) of the United States of America. The funders had no role in study design, data collection and analysis, decision to publish, or preparation of the manuscript.

2. Lu R, Zhao X, Li J, Niu P, Yang B, Wu H, et al. Genomic characterisation and epidemiology of 2019 novel coronavirus: implications for virus origins and receptor binding. Lancet Lond Engl. 2020 22;395(10224):565-74.

3. Lam TT-Y, Shum MH-H, Zhu H-C, Tong Y-G, Ni X-B, Liao Y-S, et al. Identifying SARSCoV-2 related coronaviruses in Malayan pangolins. Nature. 2020 Mar 26;1-6.

4. Andersen KG, Rambaut A, Lipkin WI, Holmes EC, Garry RF. The proximal origin of SARS-CoV-2. Nat Med. 2020 Mar 17;1-3.

5. Shu Y, McCauley J. GISAID: Global initiative on sharing all influenza data - from vision to reality. Euro Surveill Bull Eur Sur Mal Transm Eur Commun Dis Bull. 2017 30;22(13).

6. Hadfield J, Megill C, Bell SM, Huddleston J, Potter B, Callender C, et al. Nextstrain: realtime tracking of pathogen evolution. Bioinformatics. 2018 Dec 1;34(23):4121-3.

7. Peeri NC, Shrestha N, Rahman MS, Zaki R, Tan Z, Bibi S, et al. The SARS, MERS and novel coronavirus (COVID-19) epidemics, the newest and biggest global health threats: what lessons have we learned? Int J Epidemiol. 2020 Feb 22;

8. Edwards SV, Potter S, Schmitt CJ, Bragg JG, Moritz C. Reticulation, divergence, and the phylogeography-phylogenetics continuum. Proc Natl Acad Sci U S A. 2016 19;113(29):8025-32.

9. Koch H, DeGiorgio M. Maximum Likelihood Estimation of Species Trees from Gene Trees in the Presence of Ancestral Population Structure. Genome Biol Evol. 2020 Feb 1;12(2):3977-95. 
193 10. Clement M, Posada D, Crandall KA. TCS: a computer program to estimate gene genealogies. Mol Ecol. 2000 Oct;9(10):1657-9.

195

11. Leigh JW, Bryant D. popart: full-feature software for haplotype network construction. Methods Ecol Evol. 2015;6(9):1110-6.

12. Múrias dos Santos A, Cabezas MP, Tavares Al, Xavier R, Branco M. tcsBU: a tool to extend TCS network layout and visualization. Bioinformatics. 2016 Feb 15;32(4):627-8.

13. Fang B, Liu L, Yu X, Li X, Ye G, Xu J, et al. Genome-wide data inferring the evolution and population demography of the novel pneumonia coronavirus (SARS-CoV-2) [Internet]. Evolutionary Biology; 2020 Mar [cited 2020 Apr 5]. Available from: http://biorxiv.org/lookup/doi/10.1101/2020.03.04.976662

14. Yu W-B, Tang G, Zhang L, Corlett R. Decoding evolution and transmissions of novel pneumonia coronavirus (SARS-CoV-2) using the whole genomic data [Internet]. 2020. Available from: DOI: $10.12074 / 202002.00033$

15. Forster $\mathrm{P}$, Forster L, Renfrew C, Forster M. Phylogenetic network analysis of SARS-CoV2 genomes. Proc Natl Acad Sci [Internet]. 2020 Apr 8 [cited 2020 Apr 10]; Available from: https://www.pnas.org/content/early/2020/04/07/2004999117

16. Di L, Pagan PE, Packer D, Martin CL, Akther S, Ramrattan G, et al. BorreliaBase: a phylogeny-centered browser of Borrelia genomes. BMC Bioinformatics. 2014 Jul 3;15(1):233.

17. Marçais G, Delcher AL, Phillippy AM, Coston R, Salzberg SL, Zimin A. MUMmer4: A fast and versatile genome alignment system. PLoS Comput Biol. 2018;14(1):e1005944.

18. Li H, Handsaker B, Wysoker A, Fennell T, Ruan J, Homer N, et al. The Sequence Alignment/Map format and SAMtools. Bioinforma Oxf Engl. 2009 Aug 15;25(16):2078-9.

19. Bandelt $\mathrm{HJ}$, Forster $P$, Röhl A. Median-joining networks for inferring intraspecific phylogenies. Mol Biol Evol. 1999 Jan;16(1):37-48.

20. Stajich JE, Block D, Boulez K, Brenner SE, Chervitz SA, Dagdigian C, et al. The Bioperl toolkit: Perl modules for the life sciences. Genome Res. 2002 Oct;12(10):1611-8.

21. Felsenstein J. PHYLIP - Phylogeny Inference Package. Cladistics. 1989;5:164-6.

22. Camacho C, Coulouris G, Avagyan V, Ma N, Papadopoulos J, Bealer K, et al. BLAST+: architecture and applications. BMC Bioinformatics. 2009;10:421.

23. Hernández Y, Bernstein R, Pagan P, Vargas L, McCaig W, Ramrattan G, et al. BpWrapper: BioPerl-based sequence and tree utilities for rapid prototyping of bioinformatics pipelines. BMC Bioinformatics. 2018 Mar 2;19:76.

24. Edgar RC. MUSCLE: multiple sequence alignment with high accuracy and high throughput. Nucleic Acids Res. 2004;32(5):1792-7.

25. Price MN, Dehal PS, Arkin AP. FastTree 2--approximately maximum-likelihood trees for large alignments. PloS One. 2010;5(3):e9490. 
Fig 1. CoV Genome Tracker uses a maximum-parsimony mutational network (left panel) to

232 represent genealogy of SARS-CoV-2 isolates during the 2019-2020 Covid-19 pandemic. The

233 network is interactively linked with geographic origins (color-coded, top row, right) and collection

234 dates (2 ${ }^{\text {nd }}$ row, right) of viral isolates, genomic locations (at $n=146$ SNP sites) and molecular

235 nature of mutations ( $3^{\text {rd }}$ row, right), and isolate information searchable by GISAID accession (4 ${ }^{\text {th }}$

236 row, right). Colored nodes represent haplotypes $(\mathrm{n}=212)$, a unique combination of nucleotides

237 at polymorphic genome positions. Open-circle nodes $(n=4)$ represent hypothetic ancestors.

238 Each slice within a node, occupying one unit area, represents one or more viral isolates $(n=2334$

239 genomes downloaded from GISAID as of 3/29/2020) sharing a geographic origin. Thus, node

240 size is an indication of geographic diversity of a haplotype, not the number of isolates. In other

241 words, widely distributed genomes show as large nodes. Large nodes (containing >10 slices)

242 are labeled at the center. Each edge represents one or more mutational changes between a

243 parental and a descendant haplotype. Arrows indicate mutation directions determined according

244 to an outgroup genome (MN996532, strain "RaTG-13", bat icon). The network is consistent with

245 a published one consisting of half the number of genomes (15). However, the maximum parsi-

246 mony network registers 59 (or 40.7\%) sites that have changed more than once (i.e., homoplasy).

247 Causes of homoplasy include sequencing errors, presence of recombination, and the large evo-

248 lutionary distances between the outgroup and SARS-CoV-2 genomes. Nonetheless, CoV Ge-

249 nome Tracker provides up-to-date genomic changes, helps trace the origin and spread, and

250 facilitates research into virulence mechanisms and clinical interventions on the current and future

251 coronavirus outbreaks. 


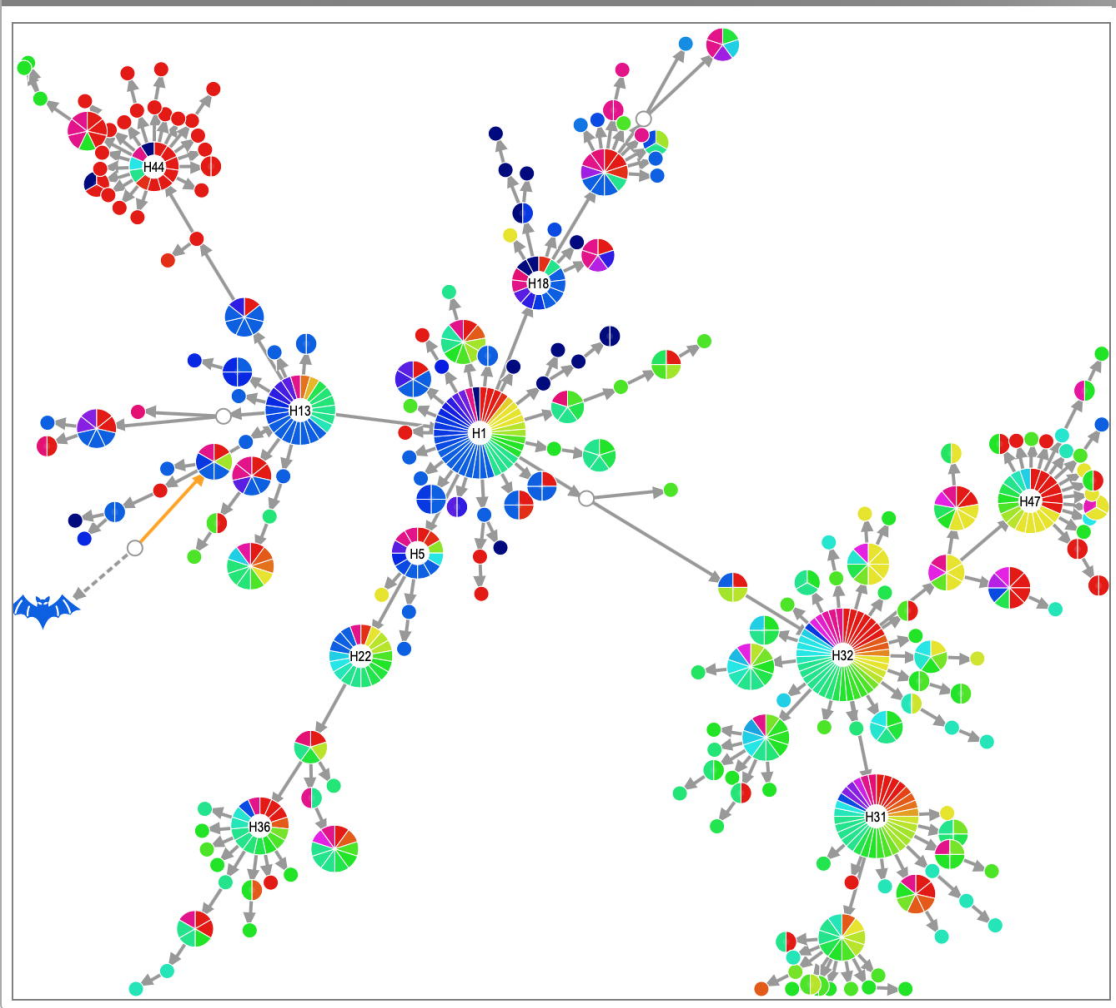

This website is maintained by Dr. Weigang Qiu's group at Hunter College of the City University of New York

Collection Site
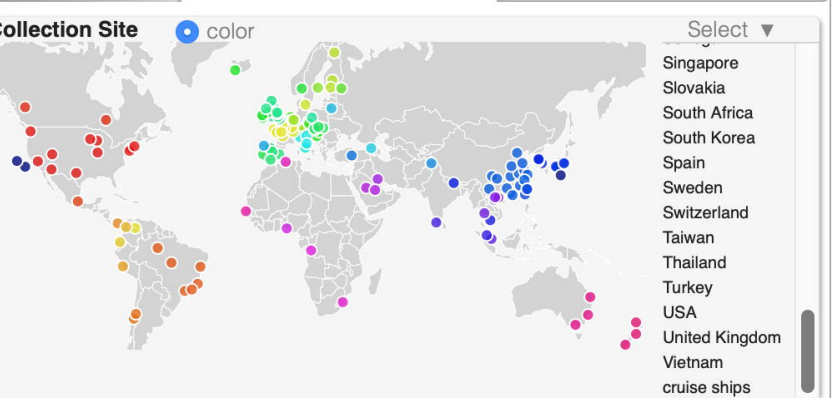

Collection Date color

01/02/20

$01 / 14 / 20 \quad 01 / 25 / 20$

$02 / 06 / 20$

$02 / 17 / 20$

02/29/20

03/12/20

$03 / 23 / 20$

\section{Genome map \& mutations}

(Wuhan-Hu-1)

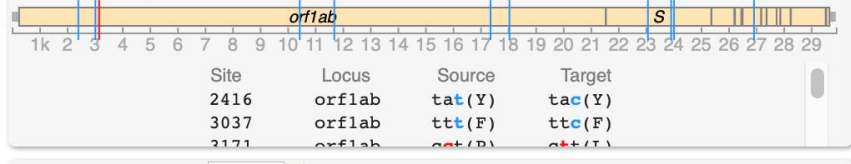

Isolate EPI_ISL_ 6 digits $\mathrm{Q}_{\text {}}$

Haplotype $\mathrm{N}=212$ a aroub of similar aenome

Isolate(s) N=2334 from GISAID
from the same location Acknowledgements
Mutation(s) at 146 genome sites
aenetic chanaes

Release 5.5 (Apr 8, 2020) 